TITLE:

\title{
Formation and collision of traveling bands in interacting deformable self-propelled particles
}

\section{$\operatorname{AUTHOR}(S):$}

Yamanaka, Sadato; Ohta, Takao

\section{CITATION:}

Yamanaka, Sadato ... [et al]. Formation and collision of traveling bands in interacting deformable self-propelled particles. Physical Review E 2014, 89(1): 012918.

ISSUE DATE:

2014-01-21

URL:

http://hdl.handle.net/2433/187275

RIGHT:

(C2014 American Physical Society 
PHYSICAL REVIEW E 89, 012918 (2014)

\title{
Formation and collision of traveling bands in interacting deformable self-propelled particles
}

\author{
Sadato Yamanaka ${ }^{1,2}$ and Takao Ohta ${ }^{1,3,4}$ \\ ${ }^{1}$ Department of Physics, Kyoto University, Kyoto 606-8502, Japan \\ ${ }^{2}$ Institute of Industrial Science, The University of Tokyo, Tokyo 153-8505, Japan \\ ${ }^{3}$ Department of Physics, The University of Tokyo, Tokyo 113-0033, Japan \\ ${ }^{4}$ Soft Matter Center, Ochanomizu University, Tokyo 112-0012, Japan
}

(Received 25 July 2013; revised manuscript received 10 October 2013; published 21 January 2014)

\begin{abstract}
We study the collective dynamics of interacting deformable self-propelled particles whose migration velocity increases with increasing local density. In two-dimensional numerical simulations of this system, the local density dependence on migration velocity leads to traveling bands similar to those previously reported for Vicsek-type models. We show that a pair of straight bands moving in opposite directions survives a head-on collision. Although traveling bands also appear in systems of constant migration velocity subjected to random noise, they are found to be unstable in a head-on collision.
\end{abstract}

DOI: 10.1103/PhysRevE.89.012918

PACS number(s): 05.45.-a, 87.18.Hf, 89.75.Fb, 05.45.Yv

\section{INTRODUCTION}

The collective dynamics of self-propelled objects have been extensively studied for almost two decades. Vicsek et al. introduced a seminal dynamical model of point particles, in which particles traveling at constant velocity align in the same direction through short-range interactions [1]. In twodimensional numerical simulations of this model with random noise, one can investigate the transition from disordered to ordered states by increasing the density of particles and/or decreasing the noise intensity. The transition properties have been investigated in detail for particles obeying the polar alignment interaction [2-6]. A coarse-grained continuum model of self-propulsion has also been introduced [7]. For more information, the reader is referred to review the articles in Refs. [8,9] and the earlier references therein.

In their numerical studies of the Vicsek-type model in two dimensions, Chaté et al. [4,10] and Bertin et al. [11] found a stable nonuniform state called a traveling band structure. The uniform ordered state destabilizes at the ordered state in the vicinity of transition point to the disordered state. With periodic boundary conditions imposed on the system, straight bands of dense, ordered particles appear in the disordered low-density matrix. On average, the particles in a band travel in a direction normal to the boundary between the ordered and disordered regions. Similar band structures have emerged in a recent model of nonpoint rigid particles with repulsive interactions [12]. Traveling concentration waves have also been observed experimentally and demonstrated numerically in dense actin motility assays [13].

The existence of traveling bands in the transition vicinity is a characteristic feature of order-disorder transitions far from equilibrium, which has no counterpart in thermal equilibrium. Traveling bands have also been investigated by a hydrodynamic description of self-propelled particles with metric alignment interactions [11,14]. These studies assume a constant migrating velocity. Another hydrodynamic approach, in which the velocity of particles increases with increasing local density, has recently yielded a similar band structure [15]. However, the opposite case, in which the migration velocity decreases with increasing local density, yields inhomogeneous structures but no traveling bands [6].

In this study, we perform a detailed particle-dynamical investigation of traveling band formation. We mainly consider that the migration velocity of individual particles increases with local density. A primary focus is the structural stability of the traveling band, originally identified in model systems of point particles. Here we account for the excluded volume and particle deformability and examine the stability of the band structure against these degrees of freedom. Furthermore, we show that these bands are robust to collision; that is, they recover from transient disturbance during collision and regain their original shape following collision.

The organization of the present paper is as follows. Section II presents our model equations that govern the interacting deformable self-propelled particles. The local-density dependence of the migration velocity is introduced. Section III presents the results of two-dimensional numerical simulations of our previous model $[16,17]$. This model investigates the effect of noise intensity on collective migration of particles moving with constant velocity. We find that traveling bands spontaneously form in simulations of large systems. In Sec. IV, the migration velocity of particles depends on the local density but the noise is removed. Numerical simulations of this scenario reveal traveling band structures in the ordered state near the order-disorder transition. The velocity of the traveling bands is theoretically treated in Sec. V. Numerical simulations of a pair of traveling bands colliding head-on are presented in Sec. VI. When the migration velocity is constant, the collision destabilizes the bands. However, when the migration velocity varies with local density, the evolved bands are robust to collisions, and they behave similarly to solitons in integrable systems. These results are discussed in Sec. VII.

\section{MODEL EQUATIONS}

We consider an assembly of interacting deformable particles in two dimensions [16,17]. For the $i$ th particle, the center of mass $r_{\alpha}^{(i)}$, velocity $v_{\alpha}^{(i)}$, and deformation tensor $S_{\alpha \beta}^{(i)}$ evolve 
in time as follows:

$$
\begin{gathered}
\frac{d}{d t} r_{\alpha}^{(i)}=v_{\alpha}^{(i)} \\
\frac{d}{d t} v_{\alpha}^{(i)}=\gamma\left(\rho^{(i)}\right) v_{\alpha}^{(i)}-\left|\mathbf{v}^{(i)}\right|^{2} v_{\alpha}^{(i)}-a S_{\alpha \beta}^{(i)} v_{\beta}^{(i)}+f_{\alpha}^{(i)}+\xi_{\alpha}^{(i)} \\
\frac{d}{d t} S_{\alpha \beta}^{(i)}=-\kappa S_{\alpha \beta}^{(i)}+b\left(v_{\alpha}^{(i)} v_{\beta}^{(i)}-\frac{1}{2}\left|\mathbf{v}^{(i)}\right|^{2} \delta_{\alpha \beta}\right)
\end{gathered}
$$

where $\kappa, a$, and $b$ are positive constants. The coefficient $\gamma$ depends on the local density as described below. The repeated Greek indices imply summation. The tensor $S$ expresses the elongation of a particle and it is represented in terms of the unit normal $\boldsymbol{n}$ parallel to the long axis of the elongated particle

$$
S_{\alpha \beta}^{(i)} \equiv s_{i}\left(n_{\alpha}^{(i)} n_{\beta}^{(i)}-\frac{1}{2} \delta_{\alpha \beta}\right),
$$

where $s_{i}(>0)$ is the magnitude of the deformation. With the terms $f_{\alpha}^{(i)}$ and $\xi_{\alpha}^{(i)}$ absent, the above set of equations describes a single self-propelled particle whose shape depends on the migration velocity [18]. The first two terms on the right-hand side of Eq. (2) where $\gamma$ is positive, make the particle travel at a constant velocity. When the particle is deformed, the velocity is modified by the third term with the coefficient $a$. In Eq. (3), the term involving the coefficient $b$ elongates the particle during its motion. When $b>0(b<0)$, elongation is parallel (perpendicular) to the migration velocity. Increasing the coefficient $\gamma$ or deceasing the values of $\kappa$ induces a bifurcation such that straight motion becomes unstable and circular motion appears [18]. In this study, we assume positive $b$ and straight motion of individual particles in the absence of interparticle interactions.

Now, we consider the interaction between a pair of particles. The term $\mathbf{f}^{(i)}$ in Eq. (2) describes the force acting on the $i$ th particle given by

$$
\mathbf{f}^{(i)}=K \sum_{j=1}^{N} \mathbf{F}_{i j} Q_{i j} .
$$

Note that the index $i$ is not included in the summation, $K$ is a positive constant, $N$ is the total number of particles, and

$$
\mathbf{F}_{i j}=-\frac{\partial U\left(\mathbf{r}_{i j}\right)}{\partial \mathbf{r}_{i j}},
$$

with $\mathbf{r}_{i j}=\mathbf{r}^{(i)}-\mathbf{r}^{(j)}$. A Gaussian potential $U\left(\mathbf{r}_{i j}\right)$ is assumed

$$
U\left(\mathbf{r}_{i j}\right)=\exp \left[-\frac{\mathbf{r}_{i j}^{2}}{2 \sigma^{2}}\right] .
$$

Throughout this paper, we take $\sigma=1$. The factor $Q_{i j}$ in Eq. (5) represents an alignment mechanism of the particles, and it is defined by

$$
Q_{i j}=1+\frac{Q}{2} \operatorname{tr}\left(S^{(i)}-S^{(j)}\right)^{2},
$$

where $Q$ is a positive constant. In two dimensions, we have

$$
Q_{i j}=1+\frac{Q}{4}\left[\left(s_{i}-s_{j}\right)^{2}+4 s_{i} s_{j} \sin ^{2}\left(\theta^{(i)}-\theta^{(j)}\right)\right]
$$

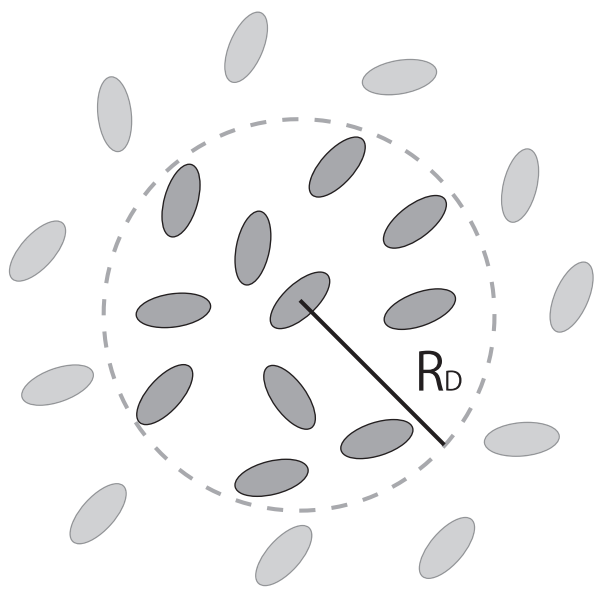

FIG. 1. Local density of the $i$ th particle defined by $N_{i} / \pi R_{D}^{2}$ where $N_{i}$ is the number of particles in the circular area of radius $R_{D}$ centered at the center of mass of the particle. The $i$ th particle is not counted in $N_{i}$.

using the parametrization $\mathbf{n}^{(i)}=\left(\cos \theta^{(i)}, \sin \theta^{(i)}\right)$. Equation (9) dictates that the repulsive interaction is weaker when two particles are elongated in parallel than when perpendicularly elongated.

The last term $\xi_{\alpha}^{(i)}$ in Eq. (2) represents Gaussian noise satisfying

$$
\begin{gathered}
\left\langle\xi_{\alpha}^{(i)}\right\rangle=0 \\
\left\langle\xi_{\alpha}^{(i)}(t) \xi_{\beta}^{(j)}\left(t^{\prime}\right)\right\rangle=\eta^{2} \delta_{i j} \delta_{\alpha \beta} \delta\left(t-t^{\prime}\right),
\end{gathered}
$$

where $\eta$ denotes the noise intensity. No fluctuation-dissipation relation is imposed since the system is out of equilibrium.

The coefficient $\gamma\left(\rho^{(i)}\right)$ in Eq. (2) indicates that the migration velocity depends on the local particle density $\rho^{(i)}$. The local density centered at the $i$ th particle is defined as

$$
\rho^{(i)}(t)=N_{i}(t) /\left(\pi R_{D}^{2}\right)
$$

where $N_{i}$ is the number of particles within distance $R_{D}$ from the center of mass of the $i$ th particle, as depicted in Fig. 1. The central $i$ th particle is excluded from the count $N_{i}$. Particle collisions introduce a temporal dependence on $N_{i}$ and hence on the the local density $\rho^{(i)}$. Here we consider that the velocity increases with increasing local density; that is, we set

$$
\gamma\left(\rho^{(i)}\right)=\gamma_{0}+\gamma_{1}\left(\frac{\rho^{(i)}(t)}{\rho_{*}}-1\right),
$$

where $\gamma_{0}, \gamma_{1}$, and $\rho_{*}$ are positive constants. Throughout the present paper, we put $\rho_{*}=0.06$. It is worth mentioning that this local density dependence on migration velocity has been observed in recent experiments of swimming bacteria [19].

To characterize the ordered and disordered states, we define the order parameter as

$$
\Phi=\left|\frac{1}{N} \sum_{j=1}^{N} e^{2 i \theta^{(j)}}\right| .
$$


Equation (14) is identical to the nematic order parameter of elongated particles. Another order parameter may be defined from the direction of migration velocity of each particle. However, we have checked that the magnitude of the alternative order parameter is not appreciably different from Eq. (14).

\section{FORMATION OF TRAVELING BANDS I}

This section presents the numerical simulations of the model system comprising Eqs. (1), (2), and (3) with constant coefficient $\gamma=\gamma_{0}$. These equations are solved on a square area with periodic boundary conditions. The parameters are fixed as $\kappa=a=\sigma=1, b=0.5, K=R_{D}=5$, and $Q=50$.

In our previous papers $[16,17]$, we obtained the phase diagram of order-disorder transitions in the space of average density $\rho_{0}$ and noise intensity $\eta$. A characteristic feature of this system is that there is a reentrant transition from an ordered state to a disordered state as the density is increased. Traveling bands emerge near this discontinuous transition in systems with sufficiently weak noises when the average density is decreased from the high-density disordered state [20]. These bands emerge only when the number of particles is very large, e.g., $N=8192$ and 32768 for the average density $\rho_{0}=0.086$ which is defined by $\rho_{0}=N /\left(L_{x} L_{y}\right) . L_{x}$ and $L_{y}$ denote the linear dimensions of the rectangular system. The properties of the band structure differ from those of ordinary bands, typically observed in Vicsek-type models [4,10,11]. In particular, the boundary of a straight band travels in a direction opposite to the migration direction of individual particles in the ordered region [20]; in ordinary bands, it travels with the migrating particles. We refer to the band, shown in Ref. [20], as an inverse band. Another kind of an inverse traveling band in which a disordered low-density stripe is surrounded by a high-density ordered state, has been reported in numerical simulations of polar disks [12]. We have carefully investigated the ordering dynamics near the reentrant transition for small values of the noise intensity. However, no ordinary bands appear as the average density is increased up to the transition threshold.

Here we investigate the behavior of the ordered state for stronger noise intensities and for a large number of the particles. Simulations were conducted with $\rho_{0}=0.03,0.04$, and 0.06, $\gamma=\gamma_{0}=1$ and $N=8192$. Traveling bands form for $\eta=0.5, \rho_{0}=0.03$, and $\eta=0.6, \rho_{0}=0.04$. (The orderdisorder transition occurs around $\eta_{c}=0.6$ for $\rho_{0}=0.03$ and $\eta_{c}=0.7$ for $\rho_{0}=0.04$.) These traveling bands are ordinary bands that travel in the direction of the individual particles. The particles outside the bands undergo noise-induced random motions. These bands do not appear in smaller systems $(N=512)$.

\section{FORMATION OF TRAVELING BANDS II}

In our previous study [17] and the present investigation of Sec. III, no ordinary traveling bands developed near the reentrant transition point when noises were very weak and $\gamma$ was independent of the local density.

Here we present the numerical results of Eqs. (1), (2), and (3) for $\gamma_{1} \neq 0$ without noise (i.e., $\eta=0$ ). The coefficients are assigned the same values as in Sec. III. In most of the

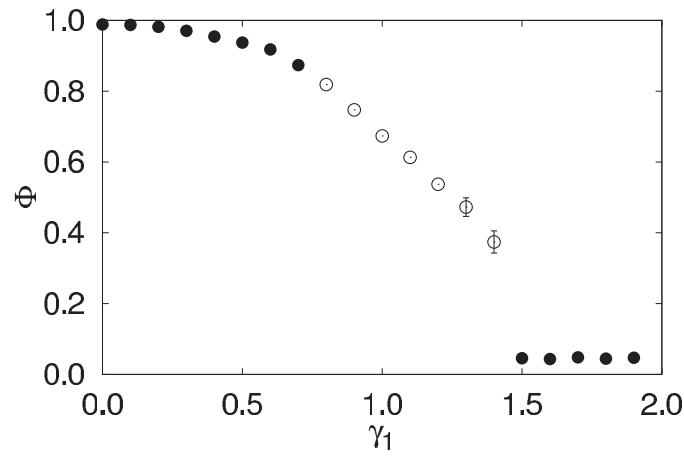

FIG. 2. Order-disorder transition as $\gamma_{1}$ is increased. Other parameters are $\gamma_{0}=0.5, N=512$, and $\rho_{0}=0.06$. The traveling bands appear at the parameters indicated by the open circles which have been obtained by five independent runs.

simulations, we specify $\rho_{0}=0.06$, and either $N=512$ or $N=8192$, to check the system size effects.

The effects by varying the coefficient $\gamma_{1}$ (with $\gamma_{0}=0.5$ and $N=512$ ) are shown in Fig. 2. Here the time-asymptotic order parameter is plotted as a function of $\gamma_{1}$. Clearly, the ordered state destabilizes when $\gamma_{1} \geqslant 1.5$. At large $\gamma_{1}$, small density fluctuations trigger the formation of high-density clusters of propagating particles, which are surrounded by almost motionless low-density particles. Since the slowly moving particles tend to be disk-shaped, the alignment mechanism (8) contained in the interaction part is ineffectively implemented, and the system becomes disordered.

The ordered state in Fig. 2 exhibits two different dynamics behaviors. When the coefficient $\gamma_{1}$ is less than 0.8 , the homogeneous ordered state emerges, in which all particles move, on average, in a certain direction. However, in the region $0.8 \leqslant \gamma_{1}<1.5$ (indicated by the open circles in Fig. 2), this homogeneous state is unstable. Traveling bands appear in which particles are propagating normal to the almost straight boundary of the band while particles outside the bands exhibit small-velocity random motions. In the small system ( $N=512$ ), a single band appears, but two or three bands are common in larger systems. A snapshot of a band structure is displayed in Fig. 3(a) where the band is traveling upward and the parameters are chosen as $\gamma_{1}=1.3, \gamma_{0}=0.5, N=8192$, and $\rho_{0}=0.06$. The density and velocity profiles are also plotted in Figs. 3(b) and 3(c), respectively. The changes of the density and the velocity are steep at the front of the band and extended at the rear. This behavior is the same as reported by Chaté et al. in their study of self-propelled point particles [4].

The shape of the ordinary traveling bands discussed at the end of Sec. III is also essentially the same as shown in Fig. 3.

The density in the disordered region can be estimated as follows. The critical local density that satisfies $\gamma=0$ is given from Eq. (13) as

$$
\rho_{c}=\rho_{*}\left(1-\frac{\gamma_{0}}{\gamma_{1}}\right) .
$$

Substituting $\gamma_{0}=0.5, \gamma_{1}=1.3$, and $\rho_{*}=0.06$ into Eq. (15), we obtain $\rho_{c}=0.037$. This result is consistent with the density $\rho \approx 0.04$ outside the band in Fig. 3(b) if we consider that $\rho_{c}$ is a sufficient condition for disordered state formation. When $\rho=$ 

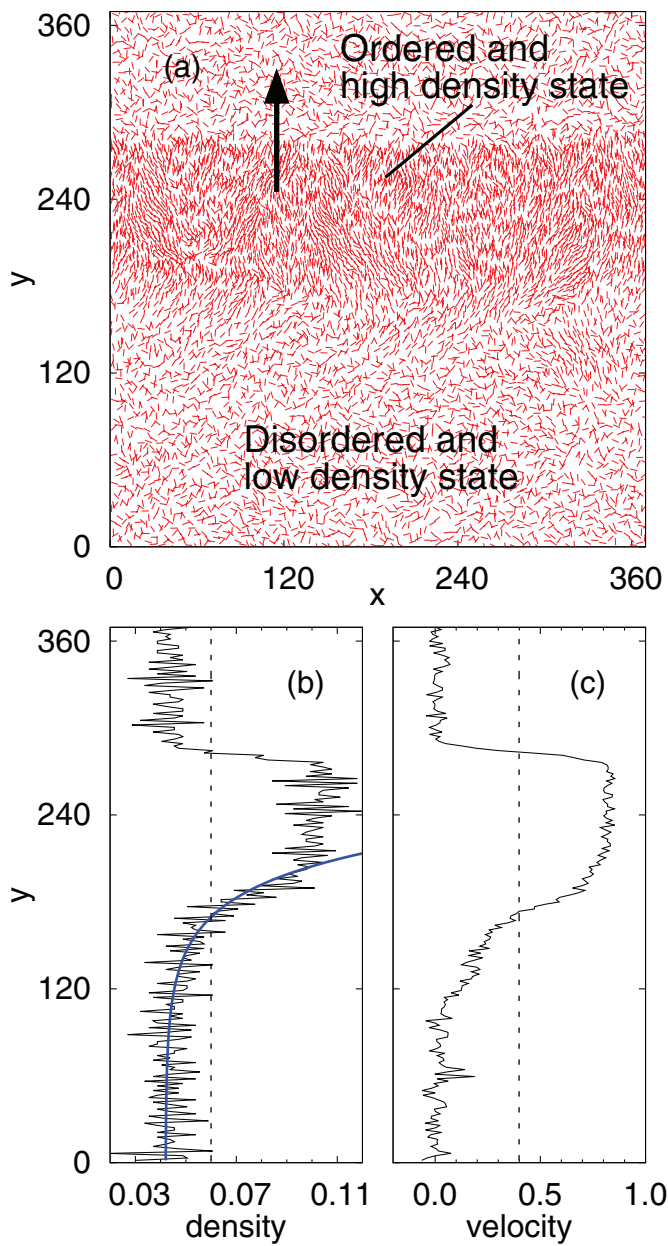

FIG. 3. (Color online) (a) An upward-traveling band (direction indicated by the arrow). Parameters are $\gamma_{0}=0.5, \gamma_{1}=1.3, N=$ 8192 , and $\rho_{0}=0.06$. Each particle is displayed as a rod emphasizing its elongated direction. (b) Density profile along the $y$ axis averaged over the $x$ direction in (a). The smooth solid line is the exponential fitting to the rear of the band. The broken line shows the average density $\rho_{0}$. (c) Velocity profile along the $y$ axis averaged over the $x$ direction in (a). The broken line indicates the $y$ component of the traveling velocity averaged over all particles.

0.04 , the average distance $\ell$ between particles is approximately $\ell=2.82$ where $\ell$ is defined by $\rho=1 /\left(\pi \ell^{2}\right)$. Therefore, the interaction potential has a factor $e^{-\ell^{2} / 2}=e^{-3.98} \approx 0.018$, sufficiently small to render the alignment ineffective.

We then examined band formation by changing the values of $\gamma_{1}$ as $\gamma_{1}=0.9,1.1$, and 1.3 while other parameters remained fixed $\left(N=512, \rho_{0}=0.06\right.$, and $\left.\gamma_{0}=0.5\right)$. Starting from random initial conditions, we carried out ten independent runs for each $\gamma_{1}$ and observed traveling bands in all simulations.

In a further series of numerical simulations, we altered the parameter $\gamma_{0}$ for fixed $\gamma_{1}$. The results for $\gamma_{1}=1.3$ are shown by the dots and the open circles in Fig. 4. For comparison, the results without the local-density dependence $\left(\gamma_{1}=0\right)$ are also plotted by the open squares. When the migration velocity is independent of the local density, the ordered state emerges for $0.2 \leqslant \gamma_{0} \leqslant 1.2$. No ordered state develops for $\gamma_{0}<0.2$ since the particle elongation induced by small migration

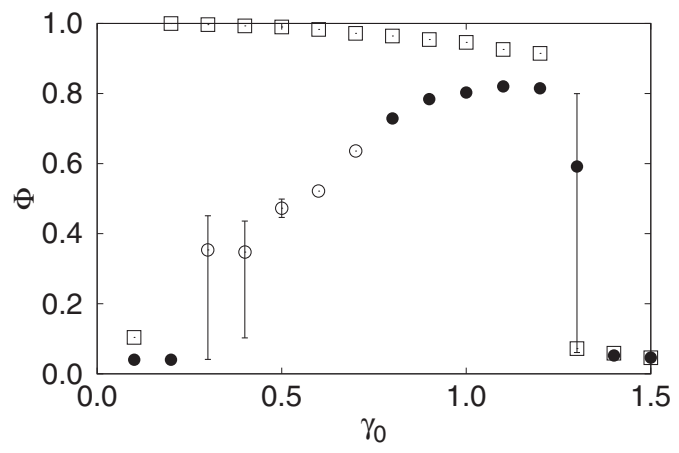

FIG. 4. Order parameter as a function of $\gamma_{0}$ for $\gamma_{1}=1.3$ (dots). Traveling bands appear in the region indicated by the open circles. For comparison, the case of no density dependence, i.e., $\gamma_{1}=0$, is also shown (open squares). The parameters are $N=512$ and $\rho_{0}=$ 0.06 . The open circles for $\gamma_{0}=0.3$ and 0.4 are the average of six independent runs while the dot for $\gamma_{0}=1.3$ has been obtained by seven runs.

velocities is insufficient to align the particles. When $\gamma_{0}$ is large, circular motion becomes relatively more stable than straight motion [18] and the alignment mechanism becomes less effective. Furthermore, when $\gamma_{0}$ is large, the kinetic energy is greater than the interaction energy, which weakens the alignment mechanism contained in the $Q$ term in Eq. (8).

This tendency also appears for $\gamma_{1}=1.3$ in Fig. 4. Note, however, that the degree of order $\Phi$ is much smaller in regions $0.3 \leqslant \gamma_{0} \leqslant 0.7$ indicated by the open circles. The homogeneous ordered state is unstable there and normal traveling bands are observed. The properties of these bands are essentially the same as those in Fig. 3.

To obtain more accurate results, numerical simulations were conducted in a large system $(N=8192)$. In this case, the maximum density inside a band does not depend strongly on the values of $\gamma_{0}$ and $\gamma_{1}$. Since the order parameter decreases as $\gamma_{1}$ increases as shown in Fig. 2, the total area covered by the bands decreases as the system approaches the order-disorder transition threshold.

\section{TRAVELING VELOCITY OF A BAND}

A theoretical derivation of the local density and velocity profiles of particles within a band, shown in Figs. 3(b) and 3(c), respectively, is beyond the scope of this study. Nonetheless, we can argue about the density-velocity relationship, based on the following continuity equation:

$$
\frac{\partial \rho(x, t)}{\partial t}=-\frac{\partial}{\partial x}(v(x, t) \rho(x, t)),
$$

where the band is moving along the $x$ axis and $\rho(x, t)=$ $\left(1 / L_{y}\right) \int_{0}^{L_{y}} d y \rho(x, y, t)$. The $x$ component $v(x, t)$ of the local particle velocity is similarly defined. We have used the approximation that $\left(1 / L_{y}\right) \int_{0}^{L_{y}} d y \rho(x, y, t) v(x, y, t) \approx \rho(x, t) v(x, t)$. By putting $\rho(x, t)=\rho(x-V t)$ where $V$ is the propagating velocity of the band, Eq. (16) is readily solved under steadystate conditions as

$$
\rho(z)=\frac{V}{V-v(z)} \rho_{\text {out }},
$$


where $z=x-V t$ and we have imposed the boundary condition $\rho \rightarrow \rho_{\text {out }}$ and $v \rightarrow 0$ for $z \pm \infty$. Therefore, setting $\rho=\rho_{f}$ and $v=v_{f}$ just at the band front, the velocity ratio is given by

$$
\frac{V}{v_{f}}=\frac{\rho_{f} / \rho_{\text {out }}}{\rho_{f} / \rho_{\text {out }}-1} .
$$

A similar relation was derived by the authors of Ref. [15]. When $\gamma_{1}=1.3$ and $N=8192$ the simulations yield, within the numerical accuracy, that $V / v_{f} \approx 1.8\left(v_{f} \approx 0.8\right)$ for all three values of $\gamma_{0}\left(\gamma_{0}=0.4,0.5\right.$, and 0.6$)$. On the other hand, the relation (18) predicts $V / v_{f} \approx 1.8$ for $\gamma_{0}=0.4$ and $V / v_{f} \approx 1.7$ for $\gamma_{0}=0.5$ and 0.6 . Here we set $\rho_{\text {out }} \approx 0.05$ and $\rho_{f} \approx 0.11$ for $\gamma_{0}=0.4$, and $\rho_{\text {out }} \approx 0.04$ and $\rho_{f} \approx 0.1$ for $\gamma_{0}=0.5$ and 0.6. The traveling band compresses the preceding particles and increases the density there, such that these particles constitute the new band front. This phenomenon explains why the front travels at higher velocity than the individual particles.

Equation (2) with $\gamma$ given by Eq. (13) indicates that the migration velocity of individual particles generally depends on $\gamma_{0}$ for fixed $\gamma_{1}$. However, as described in the previous paragraph, the values of $v_{f}$ and $V$ are found to be rather insensitive to $\gamma_{0}$. Ignoring the interaction between particles, the stationary velocity of a single particle is given by $v_{\mathrm{st}}^{2}=$ $\gamma(\rho) /(1+B)$ where $B=a b /(2 \kappa)$ [18]. Using Eq. (13) and the above values of $\rho_{f}$, we obtain $v_{\mathrm{st}}^{2} \approx 1.2,1.1$ and 1.2 for $\gamma_{0}=0.4,0.5$, and 0.6 , respectively. (The finding that $v_{\mathrm{st}}>v_{f}$ is consistent with our previous results $[16,17]$.) Since $\rho$ inside a band exhibits a weak $\gamma_{0}$ dependence and the data are scattered, we currently draw no definite conclusions about the systematic parameter dependence of $v_{f}$ and $v_{\text {st }}$.

\section{COLLISION OF TRAVELING BANDS}

To thoroughly investigate the dynamics of traveling bands, we conducted numerical simulations in a rectangular system. We first describe the traveling bands generated in a system with $\gamma_{1} \neq 0$ obtained in Sec. IV. The system size is put to be $L_{x}=640$ and $L_{y}=213.33$. Those generated for $\gamma_{1}=0$ (obtained in Sec. III) will be mentioned later in this section.

A two-band collision is displayed in Fig. 5 where $\gamma_{1}=1.1$ and $N=8192\left(\rho_{0}=0.06\right)$. Although the bands are distorted by the collision, they persist and keep traveling.

Band collision is investigated more systematically for a pair of straight bands traveling in opposite directions. These bands spontaneously develop from random initial conditions.

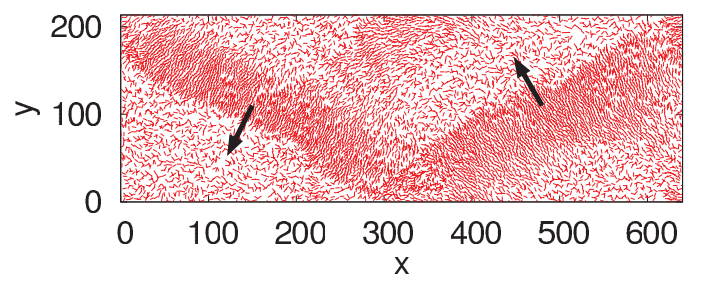

FIG. 5. (Color online) Colliding bands traveling in different directions for $\gamma_{0}=0.5, \gamma_{1}=1.1$, and $N=8192$. The arrows indicate the traveling directions. Both bands, and their traveling motions, persist after collision.

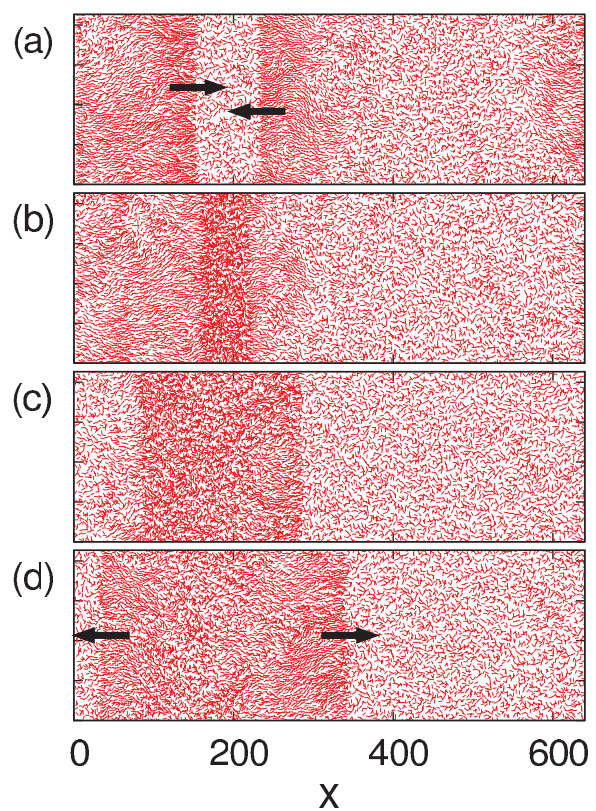

FIG. 6. (Color online) Snapshots of head-on collision of a pair of bands for $\gamma_{0}=0.5, \gamma_{1}=1.3$, and $N=8192$ at (a) $t=5636$, (b) $t=5676$, (c) $t=5716$, and (d) $t=5756$. The arrows indicate the traveling direction of the bands.

Following the initial transient, several bands appear, all traveling in the same direction. Occasionally, the largest band divides under large orientational fluctuations in the tail part, and the daughter band starts to travel in the opposite direction. Because of the periodic boundary conditions, the parent and daughter bands collide head-on. Each collision increases the size of the smaller band, until the two bands are comparable in size. The time-asymptotic behavior of the system is described below.

Time snapshots during a head-on collision where $\gamma_{0}=0.5$, $\gamma_{1}=1.3$ are displayed in Fig. 6. The orientational order is transiently destroyed in the colliding region, but the sharp fronts of a steep density gradient are preserved, as observed in Figs. 6(b) and 6(c). As the front progresses, orientational order is recovered immediately behind the front, and the bands retreat as in Fig. 6(d). A space-time plot of the local density $\rho(x, t)$ averaged along the $y$ axis is shown in Fig. 7. Clearly,

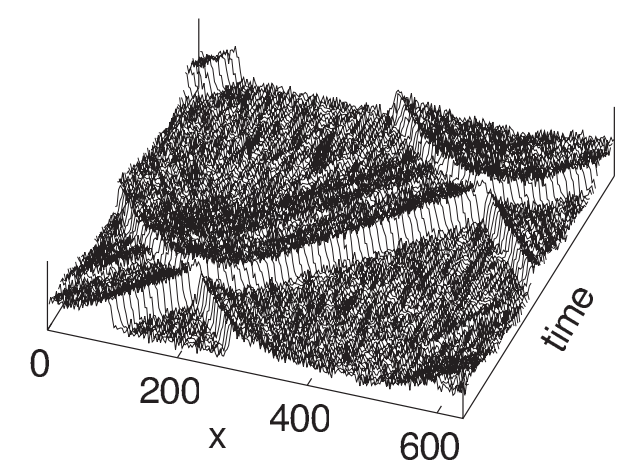

FIG. 7. Space-time profile of a pair of opposite-traveling bands. The time interval is $t=5600-6000$. Parameters are $\gamma_{0}=0.5, \gamma_{1}=$ 1.3 , and $N=8192$. 
both bands survive the collision, and the periodic boundary conditions ensure that collision survival is repeatable. We have checked that both bands retain their shape and speed after seven consecutive collisions. Following collision, the velocity of the bands is momentarily increased by the high pressure inside the colliding region, which expands the merged band. As the bands separate, their velocity again becomes constant. This soliton-like behavior of colliding traveling bands emerged for all of the investigated $\gamma_{1}\left(\gamma_{1}=0.9,1.1\right.$, and 1.3).

Next, we show the stability of traveling bands upon collisions in the systems subjected to random noises and with the constant migration velocity, i.e., $\gamma_{1}=0$. The system size is chosen as $L_{x}=783.84$ and $L_{y}=261.28$. The initial condition is a pair of oppositely traveling bands; other parameters are $\gamma_{0}=1.0, \rho_{0}=0.039$, and $N=7944$. Three independent runs were carried out at two noise intensities $\eta=0.5$ and $\eta=0.6$. In all of the runs, the traveling bands are destroyed after one or two head-on collisions, and the whole system becomes disordered. After substantial delay, a new traveling band, apparently uncorrelated to the original two bands, emerges. However, the collision behavior may depend on the aspect ratio since the lateral stability of a traveling band is expected to increase with decreasing aspect ratio. The aspect ratio of the current system is $L_{y} / L_{x}=1 / 3$.

In Ref. [4], Chaté et al. demonstrated boundary reflections of traveling bands when elastic collisions are imposed between the point particles and the boundary. We emphasize that the soliton-like behavior found for $\gamma_{1} \neq 0$ has no relation with this since the dynamics in our system are dissipative with deformability of each particle. Solitary waves have been obtained theoretically in a coarse-grained hydrodynamic model [11,21]. However, collision stability has not been investigated in this model. Quite recently, Ihle [22] adopted a kinetic approach to investigate density instability in the two-dimensional Vicsek-type model. By solving numerically the kinetic equation of the probability function that governs velocity direction and particle position, it was shown that traveling bands are not immediately destroyed by a head-on collision, but only a bigger band survives after repeated encounters. Furthermore, the properties of the band (solitary wave) seem to depend on the system size. These behaviors are inconsistent with the cases $\gamma_{1}=0$ and $\gamma_{1} \neq 0$ investigated in our model system.

\section{DISCUSSION}

We have investigated the order-disorder transitions of deformable self-propelled particles with repulsive interactions containing an alignment mechanism. A traveling band structure appears in the ordered state near the order-disorder transition point in the presence of random noises and when the magnitude of the migration velocity of particles is constant, i.e., $\gamma_{1}=0$. This result implies that traveling band structures in the Vicsek-type stochastic models [4,10,11] are structurally stable against particle deformation and the repulsive interparticle interaction. However, in the absence of noise and when the velocity of the migrating particles is independent of the local density, such ordinary band structures do not evolve. Inverse bands arise only in extremely large systems by decreasing the average density near the reentrant transition threshold [17]. By modifying the model such that the velocity of individual particles increases with increasing local density, we can generate ordinary traveling band structures even in small noiseless systems. The bands are characterized by steep frontal density and velocity profiles. In wide bands, a plateau region develops, succeeded by an exponential decay in the rear, as shown in Fig. 3.

We now present a qualitative argument for the emergence of band structures in systems whose particle velocities increase with increasing local density. Let us suppose that fluctuations generate a local high-density region. Since the migration velocity is increased in the high-density region, the particles are further elongated, enhancing their alignment in that region. The aligned particles reach the slowly moving particles ahead. Since the traveling band moves faster than individual particles, rear particles are left in the wake, and they become disordered as their density reduces. When the high-density region becomes comparable to the system size, it constitutes a traveling band because of the periodic boundary conditions. Therefore, a positive dependence of migration velocity on local density is favorable for the formation of stable traveling bands.

This qualitative explanation is consistent with recent coarse-grained hydrodynamic theories based on point particles. If the dynamics obey a Vicsek-type metric alignment interaction, the local momentum equation dictates that local velocity increases with local density, and the solutions admit traveling bands [11]. On the other hand, if the alignment interaction is topological, these terms are absent from the local momentum equation, and the homogeneous ordered state is not destabilized [23]. Introducing density-dependent migration velocity into the particle dynamics should strengthen the density-dependent terms in the coarse-grained theories.

In our system, introducing density-dependent migration velocity yielded a remarkable behavior of traveling bands, such as robustness to head-on collisions, similar to the behavior of solitons in integrable systems. In future study, we will investigate the dynamics of traveling bands in further detail by changing the parameters, for example, the softness of individual particles.

\section{ACKNOWLEDGMENTS}

We would like to thank Dr. Y. Itino for useful discussions on numerical simulations. We are grateful to an anonymous referee for useful comments which led us to additional investigations to obtain the results in Sec. III. This work was supported by Grant-in-Aids for Scientific Research A (Grant No. 24244063) and C (Grant No. 23540449) from JSPS.
[1] T. Vicsek, A. Czirók, E. Ben-Jacob, I. Cohen, and O. Shochet, Phys. Rev. Lett. 75, 1226 (1995).

[2] G. Grégoire and H. Chaté, Phys. Rev. Lett. 92, 025702 (2004).
[3] H. Chaté, F. Ginelli, G. Grégoire, F. Peruani, and F. Raynaud, Eur. Phys. J. B 64, 451 (2008).

[4] H. Chaté, F. Ginelli, G. Grégoire, and F. Raynaud, Phys. Rev. E 77, 046113 (2008). 
[5] F. Peruani, F. Ginelli, M. Bär, and H. Chaté, J. Phys.: Conf. Ser. 297, 012014 (2011)

[6] F. D. C. Farrell, M. C. Marchetti, D. Marenduzzo, and J. Tailleur, Phys. Rev. Lett. 108, 248101 (2012).

[7] J. Toner and Y. Tu, Phys. Rev. Lett. 75, 4326 (1995).

[8] T. Vicsek and A. Zafeiris, Phys. Rep. 517, 71 (2012).

[9] S. Ramaswamy, Annu. Rev. Condens. Matter Phys. 1, 323 (2010).

[10] F. Ginelli, F. Peruani, M. Bär, and H. Chaté, Phys. Rev. Lett. 104, 184502 (2010).

[11] E. Bertin, M. Droz, and G. Grégoire, J. Phys. A: Math. Theor. 42, 445001 (2009).

[12] C. A. Weber, T. Hanke, J. Deseigne, S. Léonard, O. Dauchot, E. Frey, and H. Chaté, Phys. Rev. Lett. 110, 208001 (2013).

[13] V. Schaller, C. Weber, C. Semmrich, E. Frey, and A. R. Bausch, Nature (London) 467, 73 (2010).
[14] A. Peshkov, I. S. Aranson, E. Bertin, H. Chaté, and F. Ginelli, Phys. Rev. Lett. 109, 268701 (2012).

[15] S. Mishra, A. Baskaran, and M. C. Marchetti, Phys. Rev. E 81, 061916 (2010).

[16] Y. Itino, T. Ohkuma, and T. Ohta, J. Phys. Soc. Jpn. 80, 033001 (2011).

[17] Y. Itino and T. Ohta, J. Phys. Soc. Jpn. 81, 104007 (2012).

[18] T. Ohta and T. Ohkuma, Phys. Rev. Lett. 102, 154101 (2009).

[19] A. Sokolov, I. S. Aranson, J. O. Kessler, and R. E. Goldstein, Phys. Rev. Lett. 98, 158102 (2007).

[20] M. Tarama, Y. Itino, A. M. Menzel, and T. Ohta, Euro. Phys. J. (to be published).

[21] A. Gopinath, M. F. Hagan, M. C. Marchetti, and A. Baskaran, Phys. Rev. E 85, 061903 (2012).

[22] T. Ihle, Phys. Rev. E 88, 040303(R) (2013).

[23] A. Peshkov, S. Ngo, E. Bertin, H. Chaté, and F. Ginelli, Phys. Rev. Lett. 109, 098101 (2012). 